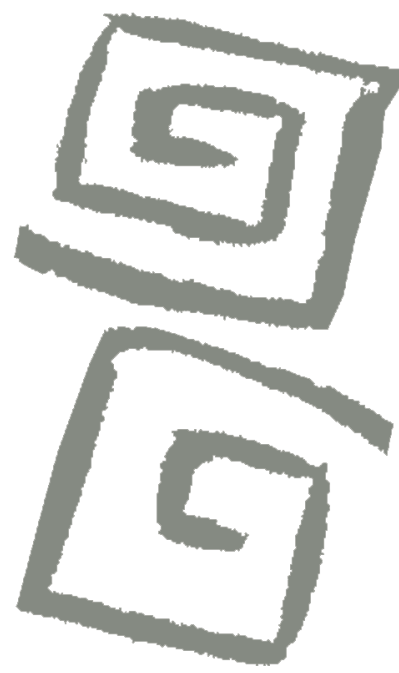

\title{
Consumo de benzodiazepinas y fármacos $Z$ en una organización de la seguridad social nacional argentina: ¿uso racional o excesivo?
}

\author{
Benzodiazepine and Z-drug consumption in a national \\ social security organization in Argentina: rational or \\ excessive use?
}

Gustavo H. Marin', Julieta del Mauro², Lupe Marin'3, Martin A. Urtasun ${ }^{4}$, Gina Marin ${ }^{5}$, Daniel Nucher ${ }^{6}$, Carlos Dacher ${ }^{7}$, Dario Diaz Perez ${ }^{8}$, Martín Cañás ${ }^{9}$

${ }^{1}$ Doctor en Medicina. Profesor Titular, Facultad de Ciencias Médicas, Universidad Nacional de la Plata Consejo Nacional le Téchicing Técnicas. Instituto Obra Social de las Fuerzas Armadas y de Seguridad Ruenos Aires, Argentina. $\bowtie$ iD

${ }^{2}$ Farmacéutica. Jefa de Unidad de Medicamentos, Instituto Obra Social de las Fuerzas Armadas y de Seguridad: Buenos Aires, Argentina. $\triangle$ (iD)

${ }^{3}$ Socióloga. Facultad de Ciencias Médicas, Universidad Nacional de la Plata, Buenos Aires, Argentina. $\square$ (iD)

${ }^{4}$ Médico. Área Farmacología, Federación Médica de la Provincia de Buenos Aires. Profesor Asociado, Universidad Nacional Arturo Jauretche, Buenos Aires, Argentina. $\bowtie$ (iD

${ }^{5}$ Licenciada en Administración. Facultad de Ciencias Médicas, Universidad Nacional de La Plata, Buenos Aires, Argentina. $\triangle$ (iD)

${ }^{6}$ Médico. Asesor, Instituto Obra Social de las Fuerzas Armadas y de Seguridad. Buenos Aires, Argentina. $\triangle$ (iD)

${ }^{7}$ Farmacéutico. Jefe de Farmacia Instituto Obra Social de las Fuerzas Armadas y de Seguridad Buenos Aires, Argentina. $M$ (iD)

${ }^{8}$ Odontólogo. Presidente, Instituto Obra Social de las Fuerzas Armadas y de Seguridad. Buenos Aires, Argentina. $₫$ (iD

${ }^{9}$ Médico. Área Farmacología, Federación Médica de la Provincia de Buenos Aires. Profesor Asociado, Universidad Nacional Arturo Jauretche, Buenos Aires, Argentina. $₫$ ii
RESUMEN Las benzodiazepinas y los "fármacos Z" (BZD-Z) se prescriben en exceso en muchos países. Este estudio evaluó su consumo en una organización de la seguridad social (obra social) de Argentina de alcance nacional. A partir de un diseño observacional descriptivo se analizó la dispensa ambulatoria de BZD-Z, entre abril 2020 y marzo 2021, a mayores de 18 años; desagregada por sexo, edad, principio activo y vida media. Se encontró una prevalencia anual de uso del 11,6\% entre los 431.445 afiliados adultos, con valores más elevados en las mujeres y mayores de 60 años. El consumo global de BZD-Z fue de 77,6 dosis diarias definidas (DDD) cada 1.000 afiliados-día. El usuario promedio recibió 5,1 dispensas anuales y el equivalente a 1,4 DDD por cada día del año. Las BZD-Z más usadas fueron las de vida media larga. El consumo de BZD-Z resultó elevado y más prolongado que lo recomendado. Es necesario mejorar la calidad en el consumo y reducir el impacto negativo del uso inapropiado de estos fármacos entre los individuos tratados.

PALABRAS CLAVES Benzodiazepinas; Utilización de Medicamentos; Farmacoepidemiología; Seguridad Social; Argentina.

\begin{abstract}
Benzodiazepines and "Z-drugs" (BZD/Z) are overprescribed in many countries. This study evaluates their consumption in a social security sector health insurance provider with national coverage in Argentina. With a descriptive and observational approach, outpatient dispensations of BZD/Zs were analyzed for people over 18 years old from April 2020 to March 2021, disaggregated by sex, age, active ingredient, and half-life. An annual prevalence of use of $11.6 \%$ was found among the 431,445 adult affiliates, with higher rates in women and in those over age 60. Overall consumption of BZD/Zs was 77.6 defined daily doses (DDD) per 1000 enrollee-days. The average user received 5.1 annual dispensations and the equivalent of 1.4 DDD for each day of the year. BZD/Zs with long half-life were the most used. We found high levels of BZD/Z consumption and for longer periods than recommended. It is necessary to improve the quality of consumption and reduce the negative impact of inappropriate use of these drugs among treated individuals. KEY WORDS Benzodiazepines; Drug Utilization; Pharmacoepidemiology; Social Security; Argentina.
\end{abstract}




\section{INTRODUCCIÓN}

Las benzodiazepinas son uno de los grupos farmacológicos más utilizados por sus efectos ansiolíticos, hipnóticos, relajantes musculares y anticonvulsivantes. Su uso se extiende con frecuencia por períodos que van más allá de los recomendados, por lo que siguen planteando controversias a nivel mundial ${ }^{(1)}$. Los llamados "fármacos Z", como zolpidem y zopiclona, estrechamente relacionados con las benzodiazepinas, fueron desarrollados como hipnóticos que no generaran tolerancia y dependencia física, pero estos mismos problemas fueron identificados al ampliar su uso ${ }^{(2)}$.

El estilo de vida imperante y las exigencias de los tiempos actuales llevan a la población a vivir situaciones cada vez más estresantes y difíciles, tanto en el marco laboral como en los propios hogares ${ }^{(3)}$. Quienes comercializan medicamentos, se han preocupado por mostrar las sustancias psicoactivas como soluciones a estas problemáticas. Es así que la población y los profesionales prescriptores relacionan las benzodiazepinas y los fármacos Z (BZD-Z) con usos diversos como dormir mejor, la reducción de la ansiedad, el aumento del rendimiento en las actividades diarias, la mejora del desempeño personal, o para mitigar situaciones sociales indeseadas, entre otros presuntos usos ${ }^{(3)}$.

El uso de este tipo de fármacos es variable según la población estudiada, y en la población adulta de Argentina varía del $4,3 \%$ al $8,1 \%{ }^{(4,5)}$; mientras que en Brasil es del 5,6\%(); ; en Uruguay del $7,4 \%^{(7)}$ y en España del $11,4 \%{ }^{(8)}$.

Otros autores advierten sobre la existencia de una gran diversidad en el consumo según el sexo, la edad, la profesión o incluso la actividad que desarrolla la población estudiada ${ }^{(9)}$. Por ejemplo, en el personal militar de la armada española, el porcentaje de consumo de BZD-Z es del $1,8 \%{ }^{(9)}$; entre el personal de enfermería este porcentaje es variable (entre un 10 y un 32\%) según el país analizado ${ }^{(10,11,12,13)} ; y$ entre otros oficios y profesiones, la variación es aún mayor ${ }^{(14)}$.

En todos los casos, la utilización indiscriminada de BZD-Z produce usuarios crónicos, susceptibles de presentar efectos de tolerancia y dependencia. Esto no ocurre generalmente cuando se prescribe para casos de ansiedad o insomnio de forma transitoria, según las pautas de la Organización Mundial de la Salud ${ }^{(15,16)}$. La suspensión brusca de los tratamientos prolongados puede provocar manifestaciones de ansiedad, ataques de pánico, hiperventilación, temblor, trastornos del sueño, espasmos musculares, anorexia, pérdida de peso, alteración visual, sudoración o disforia ${ }^{(17,18,19)}$.

Por esta razón, la duración del tratamiento debe ser lo más corta posible, aconsejándose no exceder cuatro semanas en el tratamiento del insomnio, ni más de tres meses en el de la ansiedad, incluido el tiempo de disminución de dosis hasta discontinuar la medicación ${ }^{(20)}$.

Por lo expuesto, resulta importante que cada localidad, institución o financiador de salud, conozca su realidad sobre el perfil de consumo de BZD-Z, no solo para evitar el uso innecesario de estos medicamentos, sino para prevenir los efectos indeseados asociados. Es en este marco, que los autores propusieron la realización del presente trabajo en una organización de la seguridad social (obra social) de alcance nacional, con el objetivo de conocer el uso ambulatorio de BZD-Z entre sus afiliados de 18 años y más, la cantidad y tipo de BZD-Z dispensadas durante 12 meses y el tiempo en el cual han sido utilizadas.

\section{METODOLOGÍA}

\section{Diseño y población estudiada}

Se realizó un estudio descriptivo observacional de utilización de medicamentos. La obra social tiene alcance nacional, con beneficiarios en las 24 provincias argentinas y brinda cobertura a una población de 577.000 afiliados de todos los grupos etarios, con un $20,1 \%$ de mayores de 60 años, que se desempeñan en las fuerzas armadas y de seguridad nacionales, incluyendo a los trabajadores activos, sus familiares a cargo, los jubilados y pensionados. 


\section{Fuente de datos y variables estudiadas}

La información se obtuvo de la base de datos de dispensas ambulatorias, que incluye las farmacias propias de la institución y las farmacias convenidas. Cada registro incluye la fecha de dispensa, los datos de identificación del paciente, la cantidad de envases y la composición de los fármacos dispensados.

Se analizaron las dispensas ambulatorias para la totalidad de los afiliados mayores de 18 años que consumieron medicamentos entre el $01 / 04 / 2020$ y el $31 / 03 / 2021$. Se evaluaron todas las benzodiazepinas y sus análogos con acción hipnótica (eszopiclona, zolpidem y zopiclona), en sus presentaciones como monodrogas o en combinaciones a dosis fijas (CDF).

Si bien la provisión de un medicamento no implica necesariamente su uso efectivo, en este trabajo se referirá por conveniencia como "usuario" a la persona que recibe una dispensa y se utilizará el término "afiliados" para denotar a toda la población cubierta por la obra social.

Para explorar la indicación de la BZD-Z se registró, siempre que estuviera presente, el diagnóstico consignado en la receta. Para cuantificar los diferentes fármacos con una unidad común se utilizó la dosis diaria definida (DDD), que corresponde a la dosis de mantenimiento promedio diaria de un medicamento, empleada para su indicación principal en adultos. Un comité internacional avalado por la Organización Mundial de la Salud establece el Sistema de Clasificación Anatómico, Terapéutico y Química - Anatomical Therapeutic Chemical Classification System (ATC)-, de los medicamentos y sus respectivas DDD ${ }^{(21)}$.

En el caso del clonazepam, el sistema de clasificación ATC lo ubica como anticonvulsivante y le asigna una DDD de $8 \mathrm{mg}$, acorde con esta indicación. Sin embargo, el uso predominante del clonazepam en adultos es como ansiolítico, en cuyo caso las dosis usuales son sustancialmente menores. Para reflejar apropiadamente la contribución del clonazepam a la exposición total de la población a las BZD-Z, se ha seguido la bibliografía que asigna una DDD ansiolítica de $1 \mathrm{mg}$ para este fármaco $^{(7,22,23,24)}$. En cuanto a las $C D F$, se asignaron las DDD según su componente de BZD-Z.

Se registró el sexo y la edad del usuario, el principio activo y su correspondiente código ATC, el valor de la $\mathrm{DDD}^{(21)}$ y la vida media del fármaco ${ }^{(1,25)}$. Se clasificaron las BZD-Z como de vida media corta, intermedia o larga según fuera menor de 6 horas, de 6 a 24 horas o mayor de 24 horas, respectivamente ${ }^{(25)}$.

Se cuantificaron los miligramos totales y DDD totales dispensadas en el periodo anual para cada fármaco y con esta información se determinaron las DDD de cada fármaco dispensadas diariamente cada 1.000 afiliados (DDD por 1.000 habitantes por día = DHD), mediante la siguiente fórmula ${ }^{(26)}$ :

$\mathrm{DHD}=\frac{\mathrm{mg} \text { del fármaco dispensados por año }}{\mathrm{DDD} \mathrm{mg} \times 365 \text { días } \times \mathrm{N}^{\circ} \text { de afiliados }} \times 1.000$

Se calculó la prevalencia anual de uso para cada sexo y grupo de edad, y el porcentaje de los afiliados que recibieron la dispensa de al menos un envase de BZD-Z en el período de estudio ${ }^{(27)}$. Se determinó también la cantidad promedio de BZD-Z dispensada en el año para cada usuario, expresada en envases y DDD anuales, informada también como DDD / usuario / día.

Como una aproximación a la duración y continuidad del uso, se exploró el número total de meses en los cuales a cada usuario se le dispensó al menos un envase de BZD-Z y el período máximo de dispensa ininterrumpida en meses consecutivos. Se registró la dispensa concomitante en el mismo mes de otros fármacos con potencial de interacción medicamentosas con las BZD-Z, según texto de referencia ${ }^{(28)}$.

Respecto del análisis estadístico, los datos fueron consolidados en una única base y posteriormente analizados utilizando el Software estadístico " $R$ " versión 4.0. Los datos se describen como promedios y sus desvíos estándar o porcentajes, según el tipo de variable.

Todos los datos fueron codificados para ocultar el nombre, apellido, número de afiliado y cualquier otro dato que pudiera 
identificar a los usuarios. El protocolo de estudio fue autorizado por el Comité de Ética de la Institución (D19-20).

\section{RESULTADOS}

La obra social tuvo en el período de estudio (del 01/04/2020 al 31/03/2021) un total de 431.445 afiliados mayores de 18 años, $48,3 \%$ de sexo femenino, con edad promedio de $46,5(+/-19,7)$ años.

Durante los 12 meses estudiados, se analizaron 4.833.768 dispensas, de las cuales $273.311(5,7 \%)$ correspondieron a BZD-Z, dirigidas a 50.049 usuarios diferentes $(61,9 \%$ de sexo femenino; edad promedio $64,1+/$ 16,0 años).

Los usuarios recibieron en promedio 6,6 envases anuales de BZD-Z, conteniendo en promedio 78,7 DDD por envase, resultando un total de 518 DDD anuales por usuario, lo que equivale a 1,4 DDD por día.
El porcentaje de afiliados mayores de 18 años que recibieron al menos una dispensa de BZD-Z en el lapso del año de estudio fue del $11,6 \%$. Se observó un incremento del consumo de BZD-Z a medida que aumentó la edad de los afiliados, siendo siempre mayor en el sexo femenino (Figura 1).

El promedio de la dispensa total de BZD$Z$ medido en DHD fue de 77,6 DDD diarias cada 1.000 afiliados. Las principales BZD-Z dispensadas durante el período fueron clonazepam con una DHD de 36,8 y alprazolam, con 27,6 DHD (Tabla 1). Posteriormente, siguen en orden de cantidades dispensadas el lorazepam (DHD 5,4), zolpidem (DHD 3,8), diazepam (DHD 1,8) y bromazepam (DHD $0,9)$. Las BZD-Z menos dispensadas fueron eszopiclona y zopiclona, ambas con una DHD menor a 0,1 (Tabla 1).

Las principales CDF fueron bromazepam + trimebutina, bromazepam + domperidona + simeticona, clordiazepóxido + sulpirida, alprazolam + domperidona, alprazolam + sulpirida.

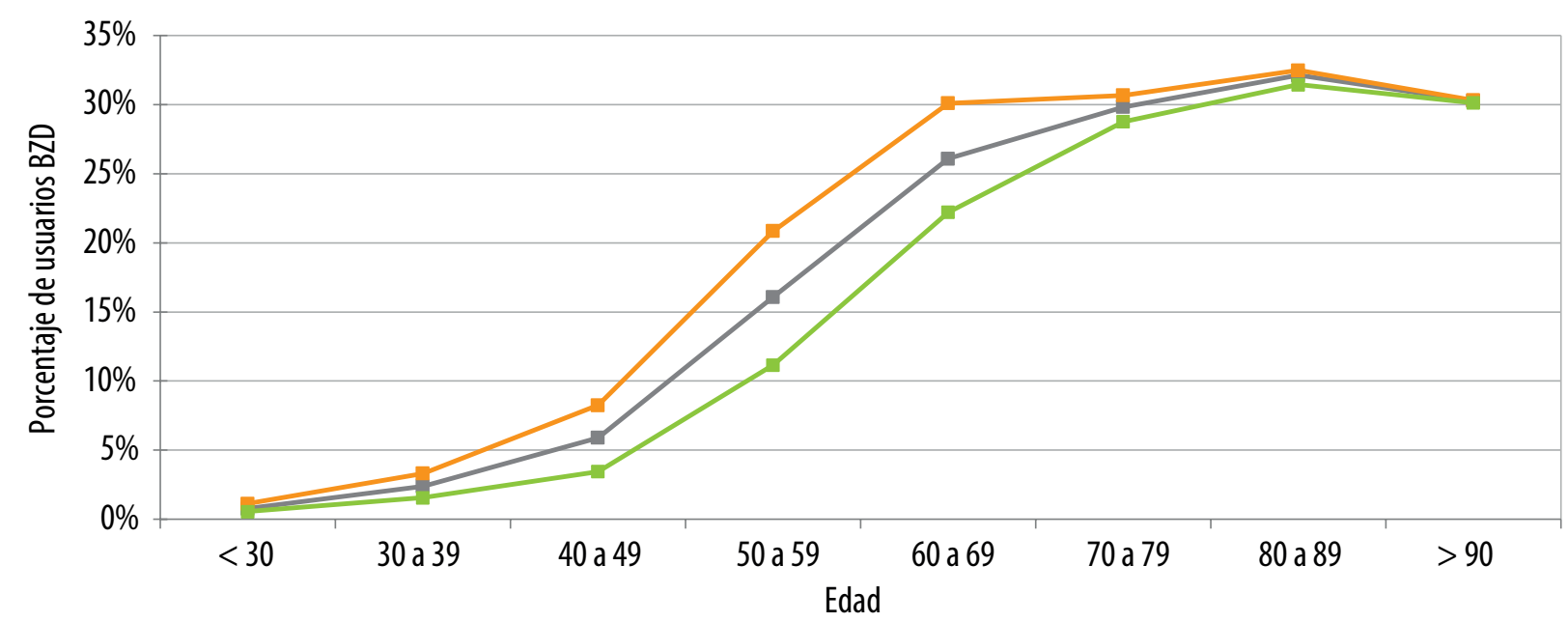

$\rightarrow$-Total usuarios BZD

Figura 1. Prevalencia anual de uso de benzodiazepinas y fármacos $Z$, según grupo etario y sexo, en una organización de la seguridad social (obra social). Argentina, 01/04/2020 al 31/03/2021.

Fuente: Elaboración propia a partir de datos provistos por el Instituto Obra Social de las Fuerzas Armadas y de Seguridad. $\mathrm{BZD}=$ Benzodiazepinas. 
Tabla 1. Dispensa de benzodiazepinas y fármacos $Z$ por parte de una organización de la seguridad social (obra social). Argentina, 01/04/2020 al 31/03/2021.

\begin{tabular}{|c|c|c|c|c|c|}
\hline Principio activo & Vida media & $\begin{array}{l}\text { DDD } \\
\text { (ATC) }\end{array}$ & $\begin{array}{l}\text { Miligramos } \\
\text { totales }\end{array}$ & $\begin{array}{c}\text { DHD } \\
\text { (DDD/1000h/día) }\end{array}$ & $\begin{array}{l}\text { Porcentaje acumulado } \\
\text { de DHD dispensadas }\end{array}$ \\
\hline Clonazepam* & Larga & 1 & 5.801 .502 & 36,84 & 47,38 \\
\hline Alprazolam & Intermedia & 1 & 4.350 .442 & 27,63 & 82,92 \\
\hline Lorazepam & Intermedia & 2,5 & 2.124 .982 & 5,40 & 89,86 \\
\hline Zolpidem & Corta & 10 & 5.917 .576 & 3,76 & 94,69 \\
\hline Diazepam & Larga & 10 & 2.891 .394 & 1,84 & 97,05 \\
\hline Bromazepam & Intermedia & 10 & 1.426 .952 & 0,91 & 98,22 \\
\hline Clobazam & Larga & 20 & 2.249 .820 & 0,71 & 99,14 \\
\hline Flunitrazepam & Intermedia & 1 & 42.021 & 0,27 & 99,48 \\
\hline Midazolam & Corta & 15 & 486.025 & 0,21 & 99,75 \\
\hline Clordiazepóxido & Intermedia & 30 & 485.025 & 0,10 & 99,88 \\
\hline Zopiclona & Corta & 10 & 131.152 & 0,08 & 99,98 \\
\hline Eszopiclona & Corta & 10 & 18.748 & 0,01 & 100,00 \\
\hline \multicolumn{6}{|c|}{$\begin{array}{l}\text { Fuente: Elaboración propia a partir de datos provistos por el Instituto Obra Social de las Fuerzas Armadas y de Seguridad. } \\
\text { DDD = dosis diarias definidas; ATC= Anatomical Therapeutic Chemical Classification System; DHD = dosis diarias definidas por } 1.000 \\
\text { habitantes por día. } \\
\text { *Sobre la DDD de } 1 \mathrm{mg} \text { de clonazepam se han seguido los trabajos de Speranza et al. }{ }^{(7)} \text {, Quaglia Planas et al. }{ }^{(22)} \text {, Zorzanelli et al. }{ }^{(23)} \text {, Kurko } \\
\text { et al. }{ }^{\left({ }^{24)} \text {. }\right.}\end{array}$} \\
\hline
\end{tabular}

Tomando en cuenta la vida media de las BZD-Z dispensadas, el $50,7 \%$ de las DDD totales correspondió a las de vida media larga, el $44,2 \%$ a las de intermedia y solo el $5,1 \%$ a las de vida media corta (Tabla 1).

Con respecto a la estimación de la duración y continuidad del tratamiento, cada usuario recibió en promedio $5,1 \pm 3,1$ dispensas en el año, con un período máximo promedio de dispensas consecutivas de 3,0 $\pm 2,4$ meses.

Los principales motivos de la prescripción fueron ansiedad $(88,4 \%)$ e insomnio $(11,6 \%)$. No obstante, los diagnósticos de estos cuadros clínicos pudieron ser registrados solo en el $24,2 \%$ de los pacientes.

Se observaron potenciales interacciones medicamentosas de las BZD-Z, incluyendo el uso concomitante con antiácidos en el 1,3\% de los casos, con antidepresivos $(1,1 \%)$, con estrógenos/progestágenos $(0,9 \%)$, con antipsicóticos $0,8 \%$, y con derivados opioides $(0,1 \%)$.

\section{DISCUSIÓN}

En una obra social argentina de alcance nacional, la dispensa total de BZD-Z, evaluada con una serie de parámetros diferentes, resultó muy elevada. El indicador más general es la exposición poblacional medida como DHD, que resultó en 77,6 DDD dispensadas diariamente cada 1.000 afiliados mayores de 18 años. Esta cantidad resultó algo inferior a las 112,4 DHD relevadas en el estudio de una obra social provincial argentina ${ }^{(29)}$ y a las 82,9 DHD consumidas en una comuna cerca de Rosario ${ }^{(22)}$. En la comparación con estudios de otros países, el valor encontrado fue apenas inferior a las registradas en los países europeos de mayor consumo como España o Portugal ( 89,3 y $96,0 \mathrm{DHD}$, respectivamente, para 2012$)^{(30,31,32)}$ y superior a lo informado en varios estudios provenientes de Australia, Costa Rica, Honduras, Dinamarca, entre

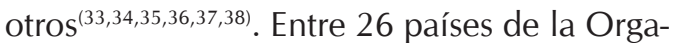
nización para la Cooperación y el Desarrollo Económicos (OCDE), ordenados por su consumo combinado de sedantes e hipnóticos, la obra social estudiada quedaría en el quinto puesto más elevado ${ }^{(39)}$.

Cabe destacar que, dado el predominio de consumidores entre los mayores de 60 años, la composición etaria de la población estudiada condiciona los resultados agregados y puede limitar la comparabilidad entre estudios. En este sentido, el 20,1\% encontrado de 
mayores de 60 años en la obra social estudiada es algo inferior al 23,1\% de América del Norte y al $25,7 \%$ de Europa ${ }^{(40)}$. De equipararse la distribución poblacional con la de estas regiones, se obtendrían en este estudio valores algo superiores a los presentados.

Al contar con información sobre los usuarios individuales se pudo calcular la prevalencia del uso de BZD-Z, cuyo valor de $11,6 \%$ es prácticamente idéntico al reportado en España ${ }^{(30)}$. Al analizarlo por sexo y edad, se comprobó que la utilización de BZD-Z fue mayor en mujeres que en hombres y que su consumo aumentaba con la edad, alcanzando su pico en los octogenarios, en concordancia con lo informado por otros autores que relevaron el consumo en diferentes países ${ }^{(41,42,43)}$.

En cuanto a la intensidad del consumo, el promedio de 1,4 DDD por usuario y por día supera claramente los valores del 1,1 del estudio argentino citado y del 0,8 de España $^{(29,30)}$. El panorama general es entonces el de una población con una alta frecuencia e intensidad de uso de estos psicofármacos.

Con relación a la selección de los fármacos utilizados, el perfil de consumo observado en el presente trabajo fue en su mayoría de BZD-Z de vida media prolongada como clonazepam, seguida de las de vida media intermedia como alprazolam, lo cual coincide con lo observado en otros trabajos de Argentina, Brasil y Uruguay $(7,22,23,29,44)$. Esta preferencia por fármacos de larga acción es contraria a las recomendaciones sobre el uso de BZD$Z$ en adultos mayores, que constituyen la mayoría de los usuarios en este estudio. Los criterios de Beers ${ }^{(45)}$ y las recomendaciones Screening Tool of Older Persons Potentially Inappropriate - Screening Tool to Alert Doctors to Right Treatment (STOPP-START)(46) señalan que implican un riesgo mayor de sedación prolongada, confusión, trastorno del equilibrio y caídas.

Las interacciones medicamentosas potenciales detectadas fueron relativamente infrecuentes. Las más habituales fueron la combinación del uso de BZD-Z con antiácidos, hormonas femeninas y antidepresivos, aspecto ya observado por otros investigadores ${ }^{(28)}$.
La administración conjunta de estos fármacos puede producir cambios en su concentración plasmática que modifiquen sus efectos.

Analizando los factores que determinan el nivel de consumo de las BZD-Z en una población, se podría postular que la diferente prevalencia de los cuadros clínicos explica las diferencias de consumo de sedantes e hipnóticos entre los países, pero la información disponible no apunta en esa dirección. Cruzando los datos de prevalencia de trastornos de ansiedad de la iniciativa Global Burden of Disease (GBD) con los de consumo de sedantes e hipnóticos de la OCDE ya citados, se observa que para 2017 la prevalencia de los cuadros de ansiedad estuvo en un rango del 3,7\% al 9,0\% (una razón de 2,5 entre los valores extremos) mientras que el consumo de BZD-Z varió entre 1,9 DHD y 110,8 DHD (un cociente de 58,3$)^{(39,47)}$. Si se toman en cuenta países como Portugal, que alcanza valores máximos de prevalencia de ansiedad y consumo de BZD-Z, se confirmaría la correlación entre patología y tratamiento. Sin embargo, países como España e Italia, con niveles de ansiedad prácticamente idénticos $(6,0$ y $6,1 \%$ respectivamente), tienen niveles de consumo de BZD-Z diametralmente opuestos (88,0 y 2,9 DHD, respectivamente) $(39,47)$. Los datos del estudio GBD informan una prevalencia de trastornos de ansiedad del 5,5\% para Argentina, mientras que los valores de 77,6 DHD encontrados en nuestro estudio, junto con lo informado en otros trabajos $^{(22,29)}$, nos ubican en un patrón de alto consumo, similar al de España.

Parece entonces que las diferencias en la prescripción de BZD-Z responden, en buena medida, a hábitos prescriptivos propios de cada sociedad. Se ha postulado que entre los determinantes podrían incluirse la capacitación de los profesionales, la organización del sistema de salud, las regulaciones legales sobre los psicotrópicos, los sistemas de cobertura de medicamentos y el grado de medicalización del malestar no patológico y de los problemas sociales ${ }^{(48)}$.

Este conjunto de factores resultará en una determinada incidencia de nuevos tratamientos con BZD-Z que, según la bibliografía 
consultada, pueden alcanzar desde el 1,2\% hasta el $7,5 \%$ de la población adulta cada año, variando según el país, el sexo y la edad de los pacientes ${ }^{(49,50,51,52)}$. Una vez iniciado el fármaco, y debido a su potencial para generar dependencia, un porcentaje de los pacientes tratados resultarán usuarios crónicos de BZD-Z, fracción estimada en un $20 \%$ en un cuidadoso estudio con 10 años de seguimiento ${ }^{(53)}$. De este modo, se va conformando año tras año la prevalencia creciente de usuarios crónicos de BZD-Z, que en nuestro estudio muestra un rápido crecimiento entre los 40 y los 60 años de edad, para alcanzar luego una meseta en las últimas décadas de la vida (Figura 1).

Desde un enfoque cualitativo, un estudio realizado en Bélgica sobre las motivaciones de los profesionales de atención primaria para iniciar un tratamiento con benzodiazepinas mostró que, aunque se perciben a sí mismos como cautelosos, prevalece la presión por ofrecer una respuesta a los problemas psicosociales de sus pacientes. Entre las razones para ello refieren la falta de propuestas alternativas y de tiempo en la consulta, considerando a las benzodiazepinas como "el mal menor", sin percibir que la naturaleza adictiva del consumo de estos fármacos sea un problema. Cabe destacar que la demanda de los pacientes no se identificó en este estudio como un factor para la prescripción ${ }^{(54)}$. En otro estudio estadounidense los médicos destacaron la eficacia y rapidez de acción de las benzodiazepinas para la ansiedad, con gran satisfacción del paciente. El uso en adultos mayores no se consideró problemático por no presentar el aumento de dosis o la conducta de búsqueda del fármaco que sugiriera adicción. Los profesionales tendían a ser escépticos sobre los riesgos del uso crónico y poco optimistas en cuanto a sus chances de lograr una disminución o suspensión del uso por parte de sus pacientes ${ }^{(55)}$.

El modo adecuado de usar las BZD-Z sigue siendo controvertido a nivel internacional, reflejando prácticas prescriptivas ampliamente diferentes. Por un lado, se resaltan los efectos adversos del uso prolongado, el componente de dependencia psicológica y física y la ausencia de estudios que respalden la eficacia del uso continuado de estos fármacos por años o por décadas ${ }^{(1,56,57)}$. Puede argumentarse que el ejemplo de los países con bajo nivel de uso de BZD-Z demuestra que una parte sustancial del uso crónico en países como el nuestro resulta innecesaria. Por otra parte, algunos autores rescatan la necesidad de dar respuesta a problemas de difícil solución, en los que el uso de psicofármacos constituiría el mal menor ${ }^{(54)}$. Otros autores destacan que, a pesar de décadas de investigación en el campo, muchos aspectos claves de la discusión aún requieren de mejores evidencias $^{(58)}$. La búsqueda de alternativas no farmacológicas eficaces para el manejo de la ansiedad y el insomnio resulta crucial en este aspecto $^{(59)}$.

\section{Limitaciones}

La duración y continuidad de los tratamientos solo pudo ser aproximada con indicadores indirectos, como el número de dispensas anuales por usuario y el período máximo de dispensas mensuales consecutivas. Estas expresan un valor mínimo para estas variables, asumiendo que cada dispensa cubre solo un mes de tratamiento. Sin embargo, dado que las dispensas alcanzan, en promedio, 78,7 DDD por envase, es de esperar que cada una de ellas cubra - para muchos usuarios- el tratamiento de más de un mes. Es sabido que el uso de BZD por más de cuatro meses es un factor de alto riesgo para el desarrollo de efectos adversos, especialmente para la población de adultos mayores ${ }^{(60,61)}$.

El período estudiado coincidió con la llegada de la pandemia de COVID-19 a la Argentina. Es interesante considerar el posible papel de las crisis económicas y sociales en el nivel de consumo de BZD-Z, teniendo en cuenta los estudios que han explorado si el aumento del estrés emocional se traduce en un mayor consumo de psicofármacos ${ }^{(62,63)}$. Sin embargo, es posible un sesgo en la dirección contraria, ya que las restricciones a la movilidad de los ciudadanos y la suspensión de las actividades no urgentes -incluida la 
atención médica habitual- pueden haber dificultado el acceso a la medicación. Al no poder comparar con datos de consumo de los años previos, el presente trabajo no permite determinar el sentido del efecto neto.

Otra limitación de este estudio fue la imposibilidad de registrar el motivo de la prescripción de BZD-Z en todos los casos, ya que el diagnóstico no es siempre requerido ni registrado en la base de dispensas de medicamentos. A partir de los datos disponibles, se observó que la ansiedad fue el principal motivo de uso, contrastando con otros estudios de Argentina donde predominó la indicación por insomnio ${ }^{(44)}$.

A partir de los resultados obtenidos en esta investigación, se desarrollaron acciones institucionales orientadas a dar racionalidad a la prescripción de BZD-Z, entre ellas intervenciones educativas destinadas a los profesionales de los prestadores propios, que incluyeron la auditoría de los tratamientos prolongados, la capacitación de prescriptores para limitar el tiempo de dispensa de
BZD-Z al período aconsejado por la OMS y la comunicación oportuna por contacto con los afiliados y profesionales de la salud para advertirles sobre potenciales interacciones medicamentosas y efectos adversos esperables por el uso de este grupo terapéutico de medicamentos.

\section{CONCLUSIÓN}

El consumo de BZD-Z en la población de afiliados de la obra social nacional estudiada resultó elevado, lo que no condice con las buenas prácticas internacionales del uso racional de este grupo de psicofármacos. Medidas correctivas implementadas a partir de los resultados obtenidos, podrán no solo controlar esta situación, sino mejorar la calidad en el consumo y reducir el impacto negativo del uso inapropiado de estos fármacos entre los individuos tratados.

\section{FINANCIAMIENTO}

Las y los autores no recibieron apoyo financiero para la investigación, autoría y/o publicación de este artículo.

\section{CONFLICTO DE INTERESES}

Las y los autores de este trabajo declaran que si bien parte del equipo de investigación trabaja en la institución que facilitó los datos analizados en este estudio, se tomaron todos los recaudos necesarios para asegurar que este vínculo no represente un conflicto de interés ni condicione el tipo de análisis y las conclusiones a las que se arriban en este artículo.

\section{REFERENCIAS BIBLIOGRÁFICAS}

1. Ashton C. Benzodiazepines: How they work \& how to withdraw - The Ashton manual. Newcastle: Institute of Neuroscience; 2002.

2. O'Donnell JM, Bies RR, Shelton RC. Tratamiento farmacológico de trastornos de depresión y ansiedad. En: Brunton LL, Hilal-Dandan R, Knollmann BC, editores. Goodman \& Gilman: Bases Farmacológicas de la Terapéutica. 13a ed. México: McGraw-Hill; 2019.
3. Lopez Vantour A, Aroche Arzuaga A, Bestard Romero J, Ocaña Fontela N. Uso y abuso de las benzodiazepinas. MEDISAN. 2010;14(4):556-560.

4. Touze G, Pawlowicz, MP, Rossi D, Goltzman P, Cymerman P. Consumo de drogas en Argentina. En: Drogas en América Latina: Estado del arte en estudios de toxicomanía en Argentina, Brasil, Colombia, Chile y Ecuador. Santiago de Chile: Ediciones Universidad Católica Silva Henríquez; 2008.

5. Brasesco MV, Legisa A, Pighin R, Tufro F. Consumo de psicofármacos y género en la Ciudad Autónoma de Buenos Aires. Buenos Aires: Observatorio de Drogas Dirección General de Políticas Sociales en Adicciones Gobierno de la Ciudad Autónoma de Buenos Aires; 2010.

6. Duarte $\mathrm{P}$, Formigoni ML. O uso de substâncias psicoativas no Brasil: módulo 1. 11a ed. Brasília: Secretaria Nacional de Políticas sobre Drogas, SUPERA; 2017.

7. Speranza N, Domínguez V, Pagano E, Artagaveytia P, Olmos I, Toledo M, Tamosiunas G. Consumo de benzodiazepinas en la población uruguaya: un posible problema de salud pública Revista Médica del Uruguay. 2015;31(2):112-119. 
8. Agencia Española de Medicamentos y Productos Sanitarios. Utilización de medicamentos ansiolíticos e hipnóticos en España durante el periodo 2000-2012: Informe de utilización de medicamentos U/HAY/V1/17012014 [Internet]. 2014 [citado 10 mar 2021] Disponible en: https://tinyurl.com/52u2zbdc.

9. González Gómez C. Prevalencia de consumo de benzodiacepinas en un grupo de población militar. Sanidad Militar. 2017;73(3):184-186.

10. Caballero Aranda I, Sevilla Lerena MP. Abuso de fármacos en medio sanitario: programas de tratamiento. Medicina y Seguridad del Trabajo. 2014;60(235):434-454.

11. Griffith J. Substance abuse disorders in nurses. Nursing Forum. 1999;34(4):19-28.

12. Trinkoff A, Zhou Q, Storr C, Soeken K. Workplace access, negative proscriptions, job stain, and substance use in registered nurses. Nursing Research. 2000;49(2):83-90.

13. Diaz CL. El uso de drogas en el personal de enfermería. Ciencia y Enfermería. 2011;17(2):37-45.

14. Observatorio Español sobre Drogas. Encuesta 20072008 sobre consumo de sustancias psicoactivas en el ámbito laboral en España [Internet]. Madrid: Ministerio de Sanidad, Política Social e Igualdad; 2014 [citado 10 mar 2021]. Disponible en: https://tinyurl.com/wjyx2b2w.

15. Organización Mundial de la Salud. Tratamiento farmacológico de los trastornos mentales en la atención primaria de salud. Washington DC: OPS; 2010. Disponible en: https://tinyurl.com/2cj8s7m8.

16. Medical \& Health Care Products Regulatory Agency. Benzodiazepines: Prescribing points [Internet]. London: MHRA; 2015 [citado 10 mar 2021]. Disponible en: https://tinyurl.com/9a2tb6z8.

17. O'Brien CP. Benzodiazepine use, abuse and dependence. Journal of Clinical Psychiatry. 2005;66(Suppl 2):28-33.

18. Smith AJ, Tett SE. Improving the use of benzodiazepines: Is it possible? A non-systematic review of interventions tried in the last 20 years. BMC Health Services Research. 2010;10:321.

19. Minaya O, Ugalde O, Fresán A. Uso inapropiado de fármacos de prescripción: dependencia a benzodiazepinas en adultos mayores. Salud Mental. 2009;32:405411.

20. Velert Vila J, Moreno Royo L, Velert Vila MM, Salar Ibáñez L. Se puede mejorar el uso de las benzodiazepinas desde la farmacia. Pharmaceutical Care España. 2012;14:94-101.

21. World Health Organization, WHO Collaborating Centre for Drug Statistics Methodology. ATC/DDD Index 2021 [Internet]. 2020 [citado 5 ago 2021]. Disponible en: https://tinyurl.com/3fjytmjt.

22. Quaglia Planas NB, Paciaroni J, Elías MM, Leiva M. Consumo de benzodiazepinas en una comuna de la región metropolitana de Rosario, provincia de Santa Fe, Argentina. Atención Primaria. 2009;41:520-521.

23. Zorzanelli RT, Giordani F, Guaraldo L, Matos GC, Brito Junior AG, Oliveira MG, Souza RM, Mota RQM, Rozenfeld S. Consumption of the benzodiazepine clonazepam (Rivotril ${ }^{\circledR}$ ) in Rio de Janeiro State, Brazil, 20092013: an ecological study. Ciência \& Saúde Coletiva. 2019;24(8):3129-3140.

24. Kurko T, Saastamoinen LK, Tuulio-Henriksson A, Taiminen T, Tiihonen J, Airaksinen M, Hietala J. Trends in the long-term use of benzodiazepine anxiolytics and hypnotics: A national register study for 2006 to 2014. Pharmacoepidemiology and Drug Safety. 2018;27(6):674-682.

25. Mihic SJ, Mayfield J, Harris RA. Hypnotics and sedatives. En: Brunton LL, Hilal-Dandan R, Knollmann BC, editors. Goodman \& Gilman's: The pharmacological basis of therapeutics. 13e ed. New York: McGraw-Hill Medical; 2018. p. 339-353.

26. Laporte JR, Tognoni G. Principios de epidemiología del medicamento. Barcelona: Masson-Salvat; 1983.

27. Hallas J, Støvring H, Pottegård A. Individual-level drug utilization analyses. En: Elseviers $M$, Wettermark B, Almarsdóttir AB, eds. Drug utilization research: Methods and Applications. New Jersey: John Wiley \& Sons; 2016. p. 68-76.

28. Moody DE. Drug interactions with Benzodiazepines. En: Mozayani A, Raymon LP, eds. Handbook of Drug Interactions: a Clinical and Forensic Guide. New York: Humana Press; 2004.

29. Cañás $M$, Marin GH, Urtasun $M$. Estudio de utilización de benzodiazepinas (BZD) en un seguro de salud provincial (IOMA) con 2 millones de beneficiarios, a partir de los datos de dispensa. XXVIII Reunión de Grupo Argentino para el Uso Racional de los Medicamentos (GAPURMED), "Situación actual de los Medicamentos: entre el conocimiento científico y las políticas sanitarias", Santa Fe - Argentina; 2019. doi: 10.13140/ RG.2.2.24788.55683.

30. Agencia Española de Medicamentos y Productos Sanitarios. Utilización de medicamentos ansiolíticos e hipnóticos en España durante el período 2000-2012 [Internet]. 2014 [citado 10 mar 2021]. Disponible en: https://tinyurl.com/m486ed2u.

31. Rayón P, Montero D, Santamaría B, Madurga M, De Abajo FJ. Benzodiazepine consumption in Spain. European Journal of Clinical Pharmacology. 1997;52:321323.

32. Furtado C. Psicofármacos: evolução do consumo em Portugal Continental $(2000$ - 2012) [Internet]. Lisboa: Infarmed, Autoridade Nacional do medicamento e produtos de saúde; 2012 [citado 10 mar 2021]. Disponible en: https://tinyurl.com/9j5zdkrm.

33. Estrela M, Herdeiro MT, Lopes Ferreira P, Roque F. The use of antidepressants, anxiolytics, sedatives and hypnotics in Europe: Focusing on mental health care in 
Portugal and prescribing in older patients. International Journal Environmental Research and Public Health. 2020;17(22):8612.

34. Islam MM, Conigrave KM, Day CA, Nguyen $\mathrm{Y}$, Haber PS. Twenty-year trends in benzodiazepine dispensing in the Australian population. Internal Medicine Journal. 2014;44(1):57-64.

35. Brandt J, Alessi-Severini S, Singer A, Leong C. Novel measures of Benzodiazepine and Z-Drug utilisation trends in a Canadian Provincial Adult Population (20012016). Journal of Population Therapeutics and Clinical Pharmacology. 2019;26(1):22-38.

36. Jiménez $M$, Claret $M$. Estudio de utilización de benzodiazepinas en el área de salud de cartago (CCSS), durante el período de febrero 2007 a enero 2008. Revista Médica de la Universidad de Costa Rica. 2009;3(1):43-55.

37. Huerta C, Abbing $\square$ Karahagopian $V$, Requena G. Exposure to benzodiazepines (anxiolytics, hypnotics and related drugs) in seven European electronic healthcare databases: a crossnnational descriptive study from the PROTECTIEU Project. Pharmacoepidemiology and Drug Safety. 2016;25(Suppl. 1):56-65.

38. Pollmann AS, Murphy AL, Bergman JC, Gardner DM. Deprescribing benzodiazepines and Z-drugs in community-dwelling adults: a scoping review. BMC Pharmacology \& Toxicology. 2015;16:19.

39. Organisation for Economic Co-operation and Development. Pharmaceutical market [Internet]. 2020 [citado 26 jun 2020]. Disponible en: https://tinyurl.com/4jj5vchz.

40. United Nations, Department of Economic and Social Affairs. World population Prospects 2019 [Internet]. 2019 [citado 26 jun 2020]. Disponible en: https://tinyurl. com/sz3pxa4e.

41. Bejarano Romero F, Lluís Piñol Moreso J, Mora Gilabert N, Claver Luquec P, Brull López N, Basora Gallisa J. Elevado consumo de benzodiazepinas en mujeres ancianas asignadas a centros de salud urbanos de atención primaria. Atención Primaria. 2008;40(12):617-621.

42. Matud Aznar MP, García Pérez L, Bethencourt Pérez JM, Rodríguez-Wangüemert C. Género y uso de medicamentos ansiolíticos e hipnóticos en España. Journal of Feminist, Gender and Women Studies. 2017;(5):23-31.

43. Torres-Bondia F, de Batlle J, Galván L, Buti M, Barbé $\mathrm{F}$, Piñol-Ripoll G. Trends in the consumption rates of benzodiazepines and benzodiazepine-related drugs in the health region of Lleida from 2002 to 2015. BMC Public Health. 2020;20(1):818.

44. Bertoldo P. Perfil del consumo de benzodiazepinas en oficinas de farmacia. Revista Cubana de Farmacia, 2019;51(4):22-32.

45. American Geriatrics Society. American Geriatrics Society 2019 updated AGS beers criteria ${ }^{\circledR}$ for potentially inappropriate medication use in older adults. Journal of the American Geriatrics Society. 2019;67(4):674-94.
46. Gallagher P, Ryan C, Byrne S, Kennedy J, O'Mahony D. STOPP (Screening Tool of Older Person's Prescriptions) and START (Screening Tool to Alert doctors to Right Treatment): Consensus validation. International Journal of Clinical Pharmacology and Therapeutics. 2008;46(2):72-83.

47. Global Burden of Disease Collaborative Network. Findings from the Global Burden of Disease Study 2017 [Internet]. Seattle: IHME; 2018 [citado 20 jun 2020]. Disponible en: https://tinyurl.com/389bp93.

48. Potočnjak I, Likić R, Degoricija V, Nham E, Wettermark B. The benzodiazepine nation of Croatia: an observational, comparative study of psychotropic drug utilization between Croatia and Sweden 2014-2015. Expert Review of Pharmacoeconomics \& Outcomes Research. 2018;18(6):641-646.

49. Alessi-Severini S, Bolton JM, Enns MW, et al. Use of benzodiazepines and related drugs in Manitoba: a population-based study. CMAJ Open. 2014;2(4):E208-E216.

50. Brett J, Karanges EA, Daniels B, Buckley NA, Schneider C, Nassir A, et al. Psychotropic medication use in Australia, 2007 to 2015: changes in annual incidence, prevalence and treatment exposure. Australian and New Zealand Journal of Psychiatry. 2017;51(10):990-999.

51. Agence Nationale de Sécurité du Médicament et des Produits de Santé. Etat des lieux de la consommation des benzodiazépines en France [Internet]. 2017 [citado 26 oct 2020]. Disponible en: https://tinyurl.com/p39aps2z.

52. Brett J, Maust DT, Bouck Z, Ignacio RV, Mecredy G, Kerr EA, Bhatia S, Elshaug AG, Pearson SA. Benzodiazepine Use in Older Adults in the United States, Ontario, and Australia from 2010 to 2016. Journal of the American Geriatrics Society. 2018;66(6):1180-1185.

53. Schonmann Y, Goren O, Bareket R, Comaneshter D, Cohen AD, Vinker $S$. Chronic hypnotic use at 10 yearsdoes the brand matter? European Journal of Clinical Pharmacology. 2018;74(12):1623-1631.

54. Anthierens S, Habraken H, Petrovic M, Christiaens T. The lesser evil? Initiating a benzodiazepine prescription in general practice: a qualitative study on GPs' perspectives. Scandinavian Journal of Primary Health Care. 2007;25(4):214-219.

55. Cook JM, Marshall R, Masci C, Coyne JC. Physicians' perspectives on prescribing benzodiazepines for older adults: a qualitative study. Journal of General Internal Medicine. 2007;22(3):303-307.

56. Lader M. Benzodiazepines revisited--will we ever learn? Addiction. 2011;106(12):2086-2109.

57. Lader M. Benzodiazepine harm: how can it be reduced? British Journal of Clinical Pharmacology. 2014;77(2):295-301.

58. Silberman E, Balon R, Starcevic V, Shader R, Cosci F, Fava GA, Nardi AE, Salzman C, Sonino N. Benzodiazepines: it's time to return to the evidence. British Journal of Psychiatry. 2021;218(3):125-127. 
59. Markota M, Rummans TA, Bostwick JM, Lapid MI. Benzodiazepine use in older adults: Dangers, management, and alternative therapies. Mayo Clinic Proceedings. 2016;91(11):1632-1639.

60. Cañás M, Urtasun M. Benzodiazepinas: Uso crónico y deprescripción. Folia Doc [Internet]. 2020;XXIII(2) [citado 10 mar 2020]. Disponible en: https://tinyurl.com/4mw4vezz.

61. García MAF, Olry de Labry Lima A, Ferrer Lopez I. et al. Analysis of changes in trends in the consumption rates of benzodiazepines and benzodiazepine-related drugs. Journal of Pharmaceutical Policy and Practice. 2018;11:1.

62. Nicieza-Garcia ML, Alonso-Lorenzo JC, Suarez-Gil $\mathrm{P}$, Rilla-Villar N. Efecto de la crisis económica sobre el consumo de psicofármacos en Asturias. Gaceta Sanitaria. 2016;30(6):464-467.

63. Colell E. Prevalencia de consumo de hipnosedantes en población ocupada y factores de estrés laboral asociados. Gaceta Sanitario. 2014;28(5):369-375.

\section{FORMA DE CITAR}

Marin GH, Del Mauro J, Marin L, Urtasun MA, Marin G, Nucher D, Dacher C, Diaz Perez D, Cañás M. Consumo de benzodiazepinas y fármacos $Z$ en una organización de la seguridad social nacional argentina: ¿uso racional o excesivo? Salud Colectiva. 2021;17:e3583. doi: 10.18294/sc.2021.3583.

Recibido: 3 may 2021 | Versión final: 23 ago 2021 | Aprobado: 9 sep 2021 | Publicado en línea: 27 sep 2021

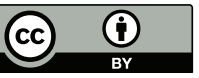

Esta obra está bajo una licencia Creative Commons Atribución 4.0 Internacional (CC BY 4.0). Atribución - Se debe dar crédito de manera adecuada, brindar un enlace a la licencia, e indicar si se han realizado cambios. Puede hacerlo en cualquier forma razonable, pero no de forma tal que sugiera que usted o su uso tienen el apoyo de la licenciante. Sin restricciones adicionales - No se pueden aplicar términos legales ni medidas tecnológicas que restrinjan legalmente a otras a hacer cualquier uso permitido por la licencia. 